\title{
Primary high grade mucoepidermoid carcinoma of skin and cervical lymphatic affection a rare entity
}

\begin{abstract}
Mucoepidermoid carcinoma is a well documented and described in the salivary glands entity, represents $20-30 \%$ of malignant tumors in minor salivary glands and multiple tumors have been reported in head and neck, paranasal sinuses, upper respiratory airways, thyroid, breast, in our experience and based on the literature, its cutaneous presentation is rare and infrequent, but identifiable by pathology and immunohistochemistry not very different from the salivary glands; Prognosis is related to histological grade, clinical outcome.
\end{abstract}

Keywords: carcinoma, metastasis, mucoepidermoid, skin

\author{
Volume 6 Issue 4 - 2018 \\ Zaki Taissoun-Aslan,' Martín Granados- \\ García,' Kuauhyama Luna-Ortiz,' Francisco \\ Javier Guerrero-Huerta,' Antonio Gómez- \\ Pedraza,' Andrea Moreno-Tobon² \\ 'Department of Head and Neck Surgery, National Cancer \\ Institute, Mexico \\ ${ }^{2}$ Department of Otolaryngology, University of Antioquia, \\ Colombia
}

Correspondence: Zaki Antonio Taissoun Aslan, Department of Head and Neck Surgery, National Cancer Institute, Mexico, Tel (+57) 300-8448804,Email info@doctorzaki.com

Received: May 20, 2018 | Published: August 03, 2018
Abbreviations: MEC, mucoepidermoid carcinoma; ASC, adenosquamous carcinoma; HPR, histopathological report; IHQ, inmunohistochemistry; PAS, periodic acid- schiff

\section{Introduction}

Mucoepidermoid carcinoma (MEC) is a well-documented and characterized pathology, being the most common tumor in the major and minor salivary glands. It is present in a $30 \%$ of all the malignant pathologies, ${ }^{1}$ which makes it a non-exclusive pathology of the head and the neck, since it can be present in the thyroid glands, breast and bronchus. ${ }^{2-5}$

Mucoepidermoid carcinoma (MEC) is referred to as an adenosquamous carcinoma (ASC). However, yet with an undefined terminology, it originates in the sweat glands in tandem with squamous and mucin producing cells, determining the differentiation between these, the histologic grade of the tumor. ${ }^{9}$

Literature tends to differentiate these two entities, basing itself on the clinic and biologic behavior. ${ }^{10-13}$ MEC is typically of a lower grade of differentiation, with lesser possibilities of presenting a locoregional or distance disease, while the high-graded ASC is a locoregional or metastatic disease in an important number of reported cases. ${ }^{14} \mathrm{~A}$ clinical case of a high-graded MEC with a metastatic locoregional disease is presented. It is treated with surgery and post surgical radiotherapy, monitoring and clinical evolution based on our institutional experience.

\section{Case report}

We are presenting a clinical case from our files, with an authorized informed consent assessed by the ethical and academic committee of the institution, a 34-year-old male identified as Mexican mestizo, with large exophytic lesion in the posterior triangle of the neck, without significant history. Physical exam: Tumor $10 \times 10 \mathrm{~cm}$, exophytic, sessile base, associated with multiple metastases in bilateral neck lymph levels II- V right tumor burden greater than $10 \mathrm{~cm}$ (Figure 1) (Figure 2). The histopathological report (HPR): poorly differentiated adenocarcinoma mucoproductor. PET/CT reports tumor metabolic activity in posterior triangle of the neck injury dependent and bilateral regional lymph node involvement with subcutaneous cellular tissue.

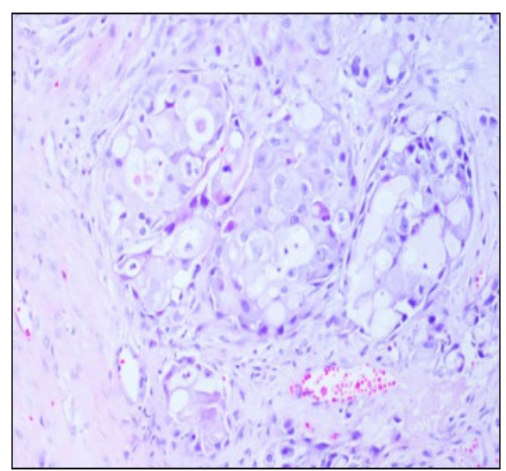

Figure I Excisional biopsy review of dermal infiltration poorly differentiated mucinous adenocarcinoma.

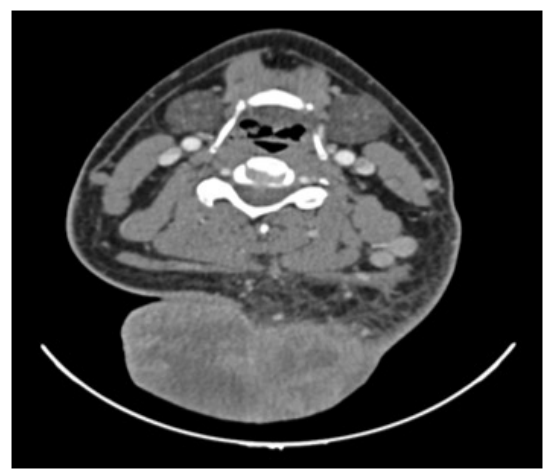

Figure 2 CT Scan, Tumor $>10 \mathrm{~cm}$ in the posterior cervical region, with affection of subcutaneous tissue.

No discarded tumor activity suggested in lung nodule surveillance (Figure 3). Surgical resection of primary lesion dimensional oncologic margins and partial thickness graft of skin. Cervical treatment was delayed until recovery program (Figure 4) (Figure 5); HPR: high-grade mucoepidermoid carcinoma with vascular lymphatic permeation and extensive areas of necrosis and perineural 
invasion. Tumor size: $14 \times 10 \times 9 \mathrm{~cm}$-free surgical margins of neoplasia; the closest to $2 \mathrm{~cm}, 2.6 \mathrm{~cm}$ surgical bed to the IHC neoplasia: PAS with and without diastase alcian blue and mucicarmine: Positive for mucin $(+++)$, cytokeratin 7, cytokeratin 8 p.63. Epithelial Membrane antigen: Positive focal $(++)$, cytokeratin 14 , cytokeratin 20 . TTF -1 : Negative (-) (Figure 6) (Figure 7).

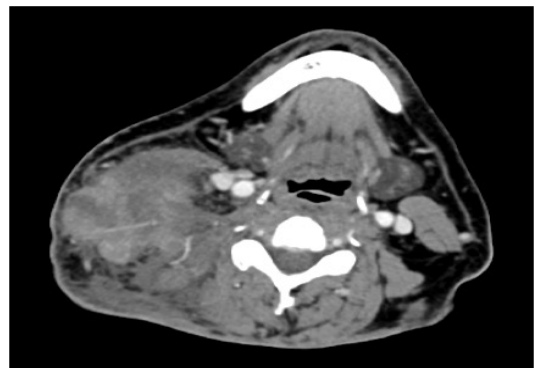

Figure 3 CT Scan, Tumor in the right posterior cervical neck region $>10 \mathrm{~cm}$ and tumor mass $14 \times 10 \times 9 \mathrm{cms}$ with thrombosis of right internal jugular vein.

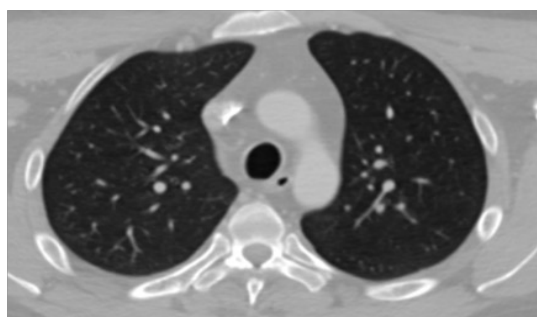

Figure 4 CT Scan. Right pulmonary apical mass, suspicious for metastatic disease $<6 \mathrm{~mm}$.

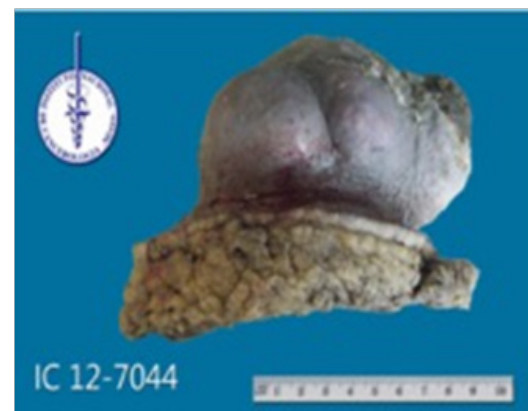

Figure 5A Three-dimensional tumor mass resected with oncological margins. Neck tumor

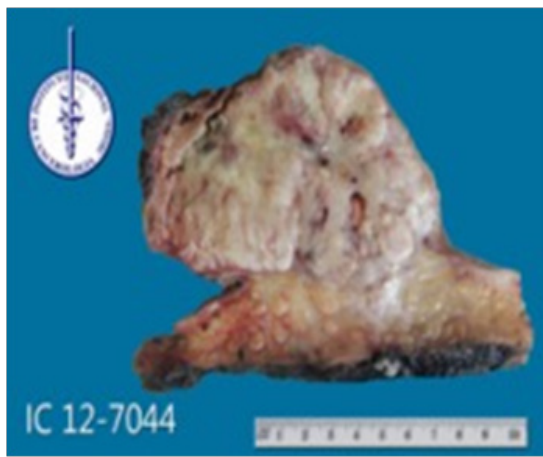

Figure 5B Three-dimensional tumor mass resected with oncological margins. Macroscopic sample.Tumor transected.

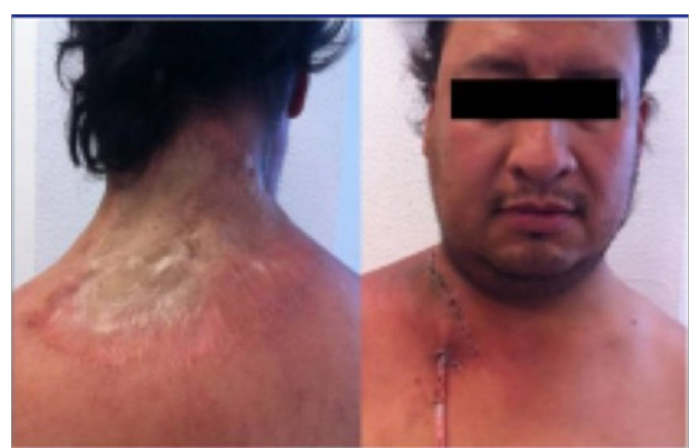

Figure 6 Postoperative patient with split thickness skin graft fully integrated.
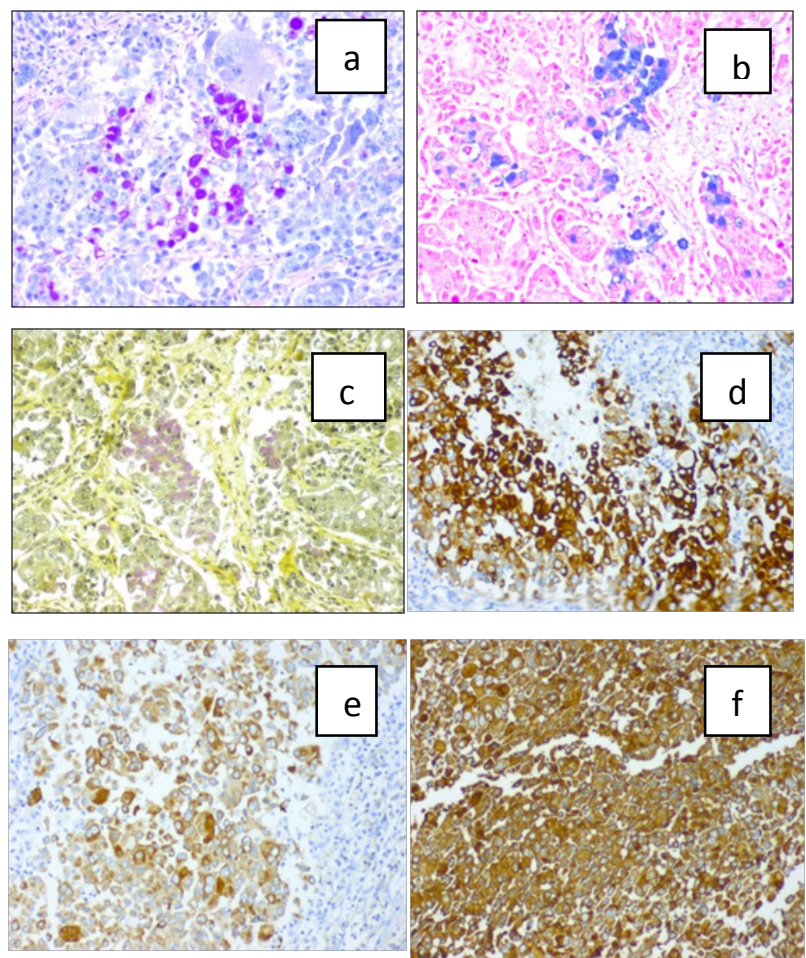

Figure $7 \mathrm{IHC}$ neoplasia : a) PAS with and without diastase, b) alcian blue and ,c) mucicarmine : Positive for mucin, d) cytokeratin 7, e) cytokeratin $8, f$ Epithelial Membrane antigen : Positive, and , cytokeratin I4, cytokeratin 20 TTF - I: were Negative.

Subsequently bilateral radical neck dissection is done $\mathrm{I}-\mathrm{Va} / \mathrm{b}$ with subsequent triangles RHP: high-grade mucoepidermoid carcinoma (dermis) and soft-tissue tumor necrosis: $40 \%$, cluster nodes with size: $7.5 \times 6 \times 5 \mathrm{~cm}$, lymphovascular permeation and invasion of perineural space, 20/20 lymph lymphoid hyperplasia. Left: lymphadenopathy 5/30 with high-grade mucoepidermoid carcinoma; submandibular glands without evidence of neoplasia. External radiation therapy is applied to cervical region $66 \mathrm{~Gy}$, currently with an overall survival was 48 months, and disease-free survival, 45 months.

\section{Discussion}

The present is a clinical case, an illustrative description of cases when clinical practice faces unusual entities and need to classify them, even when there are no antecedents. The pathology is very 
well described when it comes to salivary glands with some rare apparitions on the skin. Stewart et al. Reports that MEC is an entity with a diagnosis of histological characteristics based on the inclusion of escamous, basal and intermediate cells, a great number of musin secreting cells and an indistinguishable cellularity when it comes to a complete histological diagnosis. ${ }^{15}$ Just like MEC, ASC is more prone to appear in areas outside of the skin like the cervix, the pancreas, the lungs, the gastrointestinal tract, the head, as well as the neck. ${ }^{8,16-18}$

Clinically, the case of a 34-year-old male patient is brought forward. He presents a lesion of $<10 \mathrm{~cm}$ in the posterior triangle of the neck with cervical bilateral methastasis from the levels II-V. Initially, the pathology reported a poorly differentiated adenocarcinoma mucoproductor. Both the extension studies and the PET/CT show tumor metabolic activity in the posterior neck injury dependent and bilateral regional lymph node involvement with subcutaneous cellular tissue. Consequently, an extensive surgery with optimal oncological margins was done with immediate reconstruction and dissection of the bilateral neck. The definitive report exposes a high-grade tumor, mucoepidermoid (MEC), with preineural and linfovascular invasion. The immunohistochemistry studies show PAS with and without distase alcian blue and mucicarmine: Positive for mucin $(+++)$, cytokeratin 7, cytokeratin 8, p 63, and Epithelial Membrane antigen: Positive focal $(++)$, cytokeratin 14, cytokeratin 20, TTF -1: Negative (-). Due to the rareness of this skin neoplasm, it is necessary to perform a complete physical exam together with imaging tests with high chances of being a metastatic disease in the salivary glands. Additionally, it calls our attention the expression of the p63 with focal positivity. Recent studies suggest that the p63, member of the same genetic family than the p53, is useful in the primary or differential diagnostic of the epidermal or skin adnexal neoplasms, differentiating them from the metastatic tumors. ${ }^{19-21}$

Recent revision of MEC and ASC, of around 30 cases, show a histological difference between these clinical units (Table 1). However, MEC can appear in the form of a high-grade tumor, unlike what is presented in (Table 1). ${ }^{6}$ Approximately, 8 of the 30 documented cases were different in their biological behaviors, with high grade histologies, locoregional dissemination, metastasic and death. ${ }^{22,23}$

Table I Histological characteristics of the mucoepidermoid carcinoma (MEC) and adenosquamous carcinoma (ASC)

\begin{tabular}{lll}
\hline & MEC & ASC \\
\hline Intraepidermal carcinoma & - & + \\
Well-differentiated adenocarcinoma & - & + \\
Dermal -based solid /cystic tumor & + & - \\
Papillary features & + & - \\
Mucigenic cell & ++ & - \\
Peritumoral fibrosis & + & - \\
High-grade nuclear features & - & $-/+$ \\
\hline
\end{tabular}

Keyvan $\mathrm{N}$ et al. makes reference to the successful use of the mohs micrographic surgery in a high histological grade MEC with $1.5 \mathrm{~cm}$ margins and to the oncoplastic reconstruction with a global survival rate of 36 months without postoperatoy radiotherapy. ${ }^{24}$ This sounds almost anecdotic, since a high-grade (MEC) is known to be very aggressive. In our report, we describe the case of a large tumor located in the posterior triangle of the neck with a bilateral locoregional disease and without metastatic disease, present in a 50\% of the cases, mainly in the lungs. ${ }^{2}$ The standard treatment for MEC is a surgery with acceptable oncological margins, with or without dissection of the neck, followed by an oncoplastic reconstruction (if needed). The postoperatory external radiotherapy or intensity- modulated radiotherapy in doses of 60-70 Gy in intermediate graded and high graded tumors, ${ }^{2}$ with recurrences vary between $30-50$ and $12 \%$ of the high graded tumors, successively lesser in the low graded ones. ${ }^{10}$ Our actual experience proves that the global survival rate was of 48 months, currently under monitoring, and the survival rate without the disease is of 45 months.

It is extremely difficult to know which the ideal treatment for MEC is, due to the fact that the published experiences belong to retrospective studies of the tumors in the salivary glands. Plus, in a series of primarily skin MEC cases, we consider that the experience of the institution is key in the curative oncological treatment, based on their experiences and multidisciplinary medical sessions to define a treatment plan.

\section{Conclusion}

Mucoepiedermoid skin carcinoma with lymph node metastasis is extremely rare, its clinical Figure depends obviously from the source site, can be classified into low, intermediate, and high grade which is composed of poorly differentiated tumors with mucin secreting cells, cells squamous and intermediate cells. It is generally accepted that the treatment of choice in resectable lesions is surgery with acceptable oncological margins, accompanied by radiotherapy as adjuvant treatment to avoid recurrence and disease control, but also in high-grade tumors, however in the literature currently there is no consensus on the appropriate treatment, based only on case reports and experiences of institutions.

\section{Acknowledgements}

None.

\section{Conflict of interest}

Author declares that there is no conflict of interest.

\section{References}

1. Ellis GL, Auclair PL. Tumors of the Salivary Gland. Washington DC: Armed Forces Institute of Pathology; 1996:155-176.

2. Baloch ZW, Solomon AC, LiVolsi VA. Primary mucoepidermoid carcinoma and sclerosing mucoepidermoid carcinoma with eosinophils of the thyroid gland: a report of nine cases. 2000;13(7):802-7.

3. Kim TS, Lee KS, Han J, et al. Mucoepidermoid carcinoma of the tracheobronchial tree: radiographic and CT findings in 12 patients. Radiology. 1999;212(3):643-8.

4. Foschini PM, Filho RJS, Eusebi V,et al. Salivarygland-like tumours of the breast: Surgical and molecular pathology. J Clin Pathol. 2003;56(7):497-50.

5. Simpson RJ, Hoang KG, Hyams VJ, et al. Mucoepidermoid carcinoma of the maxillary sinus. Otolaryngol Head Neck Surg. 1988;99(4):419-23.

6. Yen A, Sanchez RL, Fearneyhough P, et al. Mucoepidermoid carcinoma with cutaneous presentation. JAAD. 1997;37(2 Pt 2):340-342.

7. Landman G, Farmer ER. Primary cutaneous mucoepidermoid carcinoma: report of a case. J Cutan Pathol. 1991;18(1):56-9.

8. Friedman KJ. Low-grade primary cutaneous adenosquamous (mucoepidermoid) carcinoma. Am J Dermatopathol. 1989;11(1):43-50.

9. Gallagher HS, Miller GV, Grampa G. Primary mucoepidermoid carcinoma of the skin. Cancer. 1959;12:286-288. 
10. Gerughty RM, Hennigar GR, Brown FM. Adenosquamous carcinoma of the nasal, oral and laryngeal cavities, a clinico- pathologic survey of ten cases. Cancer. 1968;22(6):1140-55.

11. Azorín D, López-Ríos F, Ballestín C, et al. Primary cutaneous adenosquamous carcinoma: a case report and review of the literature. $J$ Cutan Pathol. 2001;28(10):542-545.

12. Banks ER, Cooper PH. Adenosquamous carcinoma of the skin: a report of 10 cases. J Cutan Pathol. 1991;18(4):227-34.

13. Farley JH, Hickey KW, Carlson JW, et al. Adenosquamous histology predicts a poor out- come for patients with advanced-stage, but not early-stage. Cancer. 2003;97(9):2196-202.

14. Minni A, Roukos R, De Carlo A, et al. Primary cutaneous mucoepidermoid carcinoma infiltrating the parotid gland. Eur Rev Med Pharmacol Sci. 2012;16 Suppl 4:26-29.

15. Stewart FW, Foote FW, Becker WF. Muco-epidermoid tumors of salivary glands. Ann Surg. 1945;122:820-844.

16. Johnson DS, Solomon AR, Washington CV. Mucoepidermoid/adenosquamous carcinoma of the skin: presentation of two cases. Dermatol Surg. $1945 ; 122(5): 820-844$.

17. Nouri K, Trent JT, Lowell B, et al. Mucoepidermoid carcinoma (adenosquamous carcinoma) treated with Mohs micrographic surgery.
Int J Dermatol. 2003;42(12):957-859.

18. Vogel MH. Mucoepidermoid carcinomas of the lid: a clinico-pathologic report of two cases. Ophthalmologica. 977;174(3):171-175.

19. Ivan D, Hafeez Diwan A, Prieto VG. Expression of p63 in primary cutaneous adnexal neoplasms and adenocarcinoma metastatic to the skin. Mod Pathol. 2005;18(1):137-42.

20. Ivan D, Nash JW, Prieto VG, et al. Use of p63 expression in distinguishing primary and metastatic cutaneous adnexal neoplasms from metastatic adenocarcinoma to skin. J Cutan Pathol. 2007;34(6):474-480.

21. Suarez PAJM,Vieites B,ErnestinaE, et al. Primarymucoepi-dermoid carcinoma of the skin expressing p63. Am J Am J Dermatopathol. 2010;32(1):61-64.

22. Locati LD, Quattrone P, Pizzin N, et al. Primary high-grade mucoepidermoid carcinoma of the minor salivary glands with cutaneous metastases at diagnosis. Oral Oncol. 2002;38(4):401-404.

23. Smoller BR, Narurkar V. Mucoepidermoid carcinoma metastatic to the skin: an histologic mimic of a primary sweat gland carcinoma. $J$ Dermatol Surg Oncol. 1992;18(5):365-368.

24. Keyvan Nouri MD, Jennifer T, Trent MD. Mucoepidermoid carcinoma (adenosquamous carcinoma) treated with Mohs micrographic surgery. International Journal of Dermatology. 2003;42(12):957-959. 